\title{
An Unavoidable Challenge for Ni-rich Positive Electrode Materials for Lithium-Ion Batteries
}

Hongyang Li a , Aaron Liu ${ }^{b}$, Ning Zhang a,c , Yiqiao Wang $\stackrel{d}{\text {, Shuo Yin }} \stackrel{\text { d, Haohan Wu }}{\text { d,e }}{ }^{\text {and Jeff }}$ R. Dahn *,a

a Physics and Atmosphere Science, Dalhousie University, Halifax, NS B3H 3J5, Canada

${ }^{\mathrm{b}}$ Department of Chemistry, Dalhousie University, Halifax, NS B3H 4R2, Canada

c School of Metallurgy, Northeastern University, Shenyang 110819, China

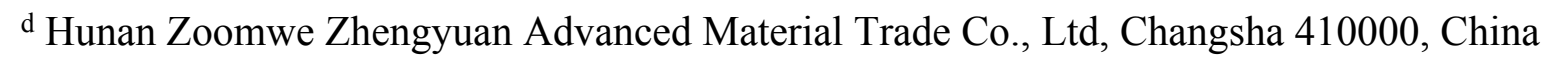

e Hoffmann Institute of Advanced Materials, Shenzhen Polytechnic, Shenzhen 518000, China

*corresponding author - jeff.dahn@dal.ca. Tel.: 001-902-494-2991; Fax: 001-902-494-5191 
Figure S1

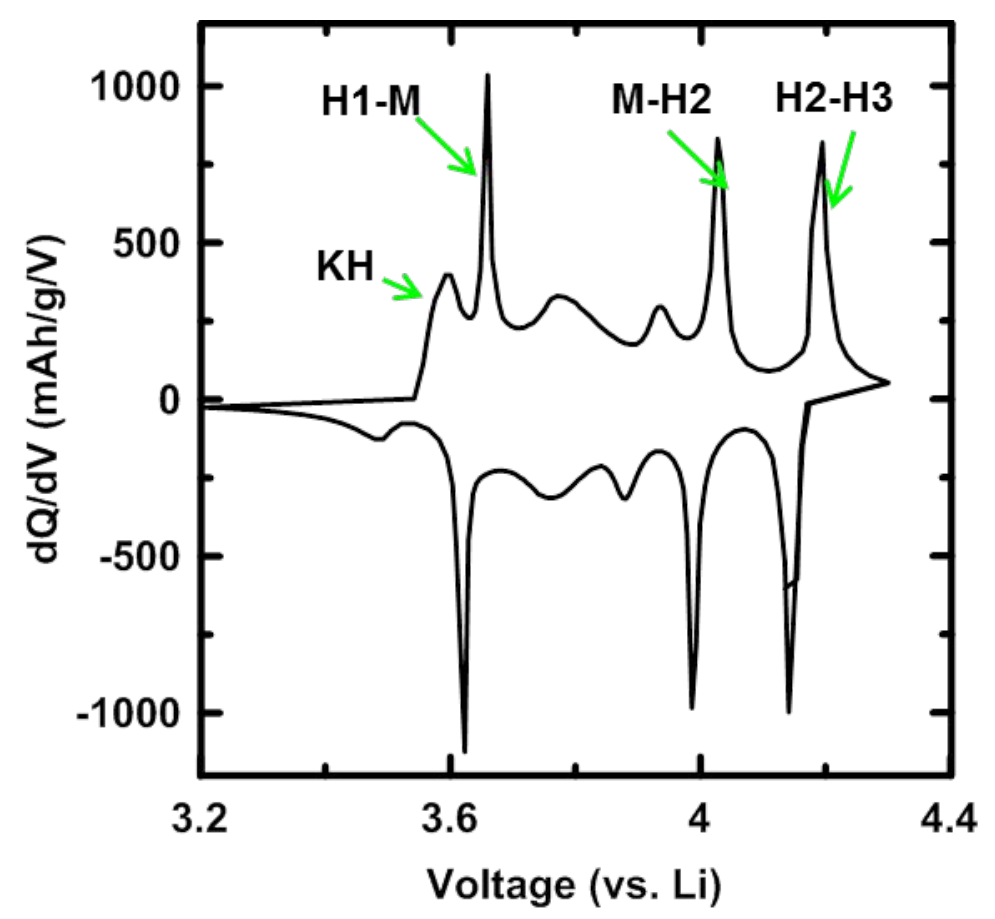

Figure S1. Differential capacity vs. voltage (dQ/dV vs. V) of $\mathrm{LiNiO}_{2}$ with the kinetic hindrance $(\mathrm{KH})$ region and phase transition peaks marked. 
Figure S2
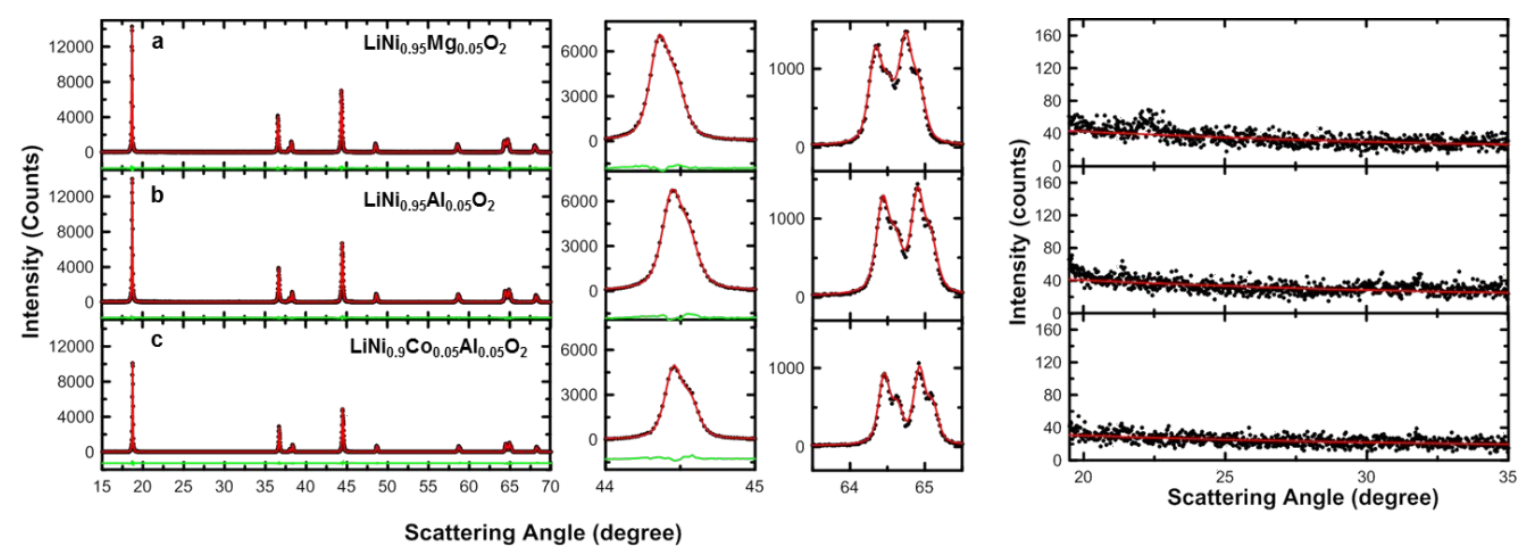

Figure S2. XRD patterns of (a) $\mathrm{LiNi}_{0.95} \mathrm{Mg}_{0.05} \mathrm{O}_{2}$, (b) $\mathrm{LiNi}_{0.95} \mathrm{Al}_{0.05} \mathrm{O}_{2}$, and (c) $\mathrm{LiNi}_{0.9} \mathrm{Co}_{0.05} \mathrm{Al}_{0.05} \mathrm{O}_{2}$. Expanded view of the (104) Bragg peak, (108)/(110) Bragg peaks, and impurity regions were shown. Black circles are experimental XRD data, solid red lines are calculated patterns from Rietveld refinement, and green lines show the differences between the measured and calculated patterns. 
Figure S3
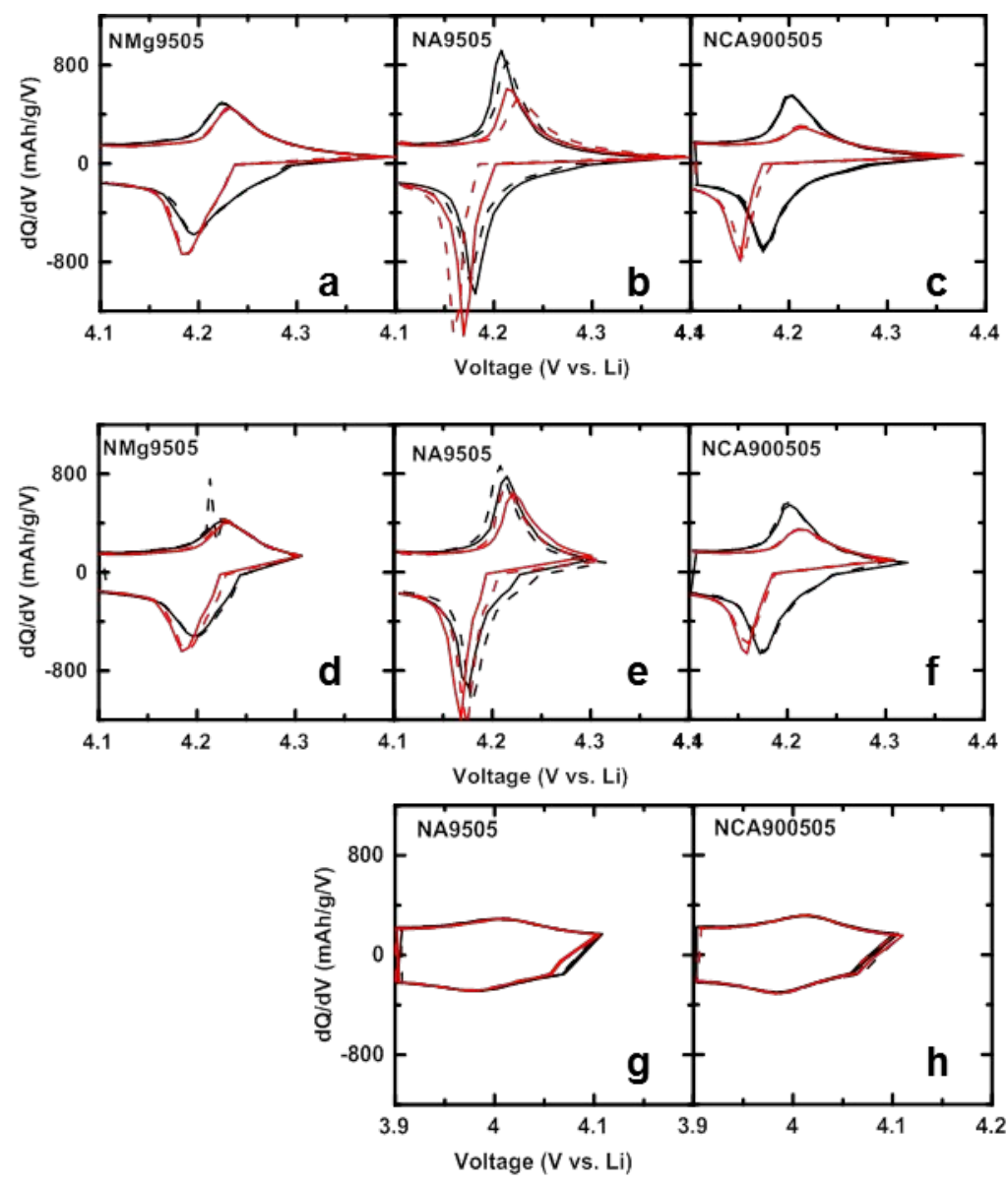

Figure S3. $(\mathrm{a}-\mathrm{h})$ The expanded views of $\mathrm{dQ} / \mathrm{dV}$ vs. $\mathrm{V}$ of the $1^{\text {st }}$ (black) and $50^{\text {th }}$ (red) cycles for the data shown in Figure 5. Solid and dashed lines in the same color represent pair cells. 
Figure S4.
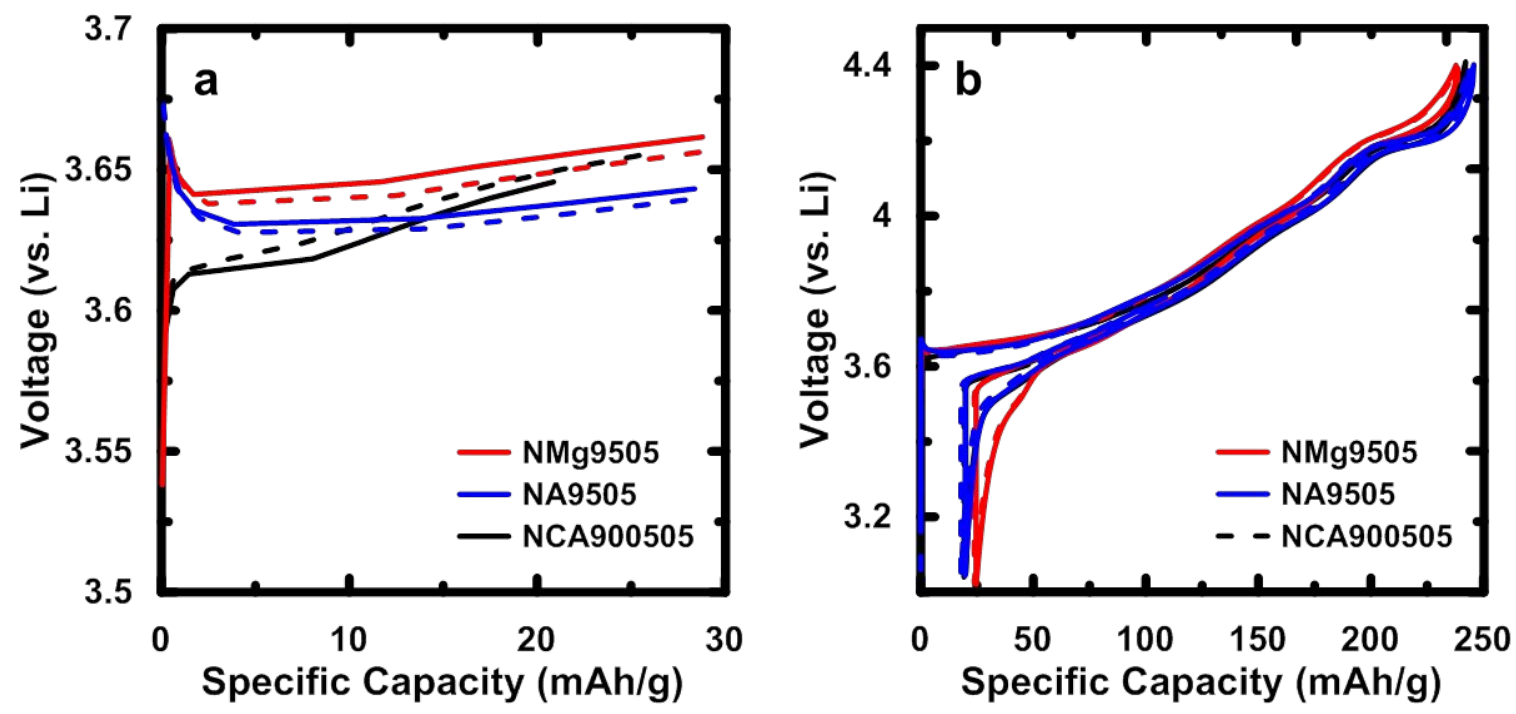

Figure S4. (a) Blow-up view of the $1^{\text {st }}$ cycle voltage profiles for NMg9505, NA9505, and NCA900505 half cells. Solid and dashed curves represent pair cells. (b) Full view of the voltage profile. 